\title{
Impacto económico del sector cerámico en San José de Cúcuta (Colombial $^{*}$
}

\section{Economic Impact of the Ceramic Sector on San Jose de Cucuta (Colombia)} Impacto económico do setor cerâmico em San
José de Cúcuta (Colômbia)

\author{
Johanna Milena Mogrovejo Andrade ${ }^{* \star}$ \\ Liliana Marcela Bastos Osorio ${ }^{\star \star \star}$ \\ Jhon Antuny Pabón ${ }^{* \star *}$
}

\footnotetext{
Este artículo presenta los resultados del proyecto de investigación titulado "Impacto económico del sector cerámico en la ciudad de San José de Cúcuta". El objetivo general de este proyecto es "determinar el impacto económico de las empresas del sector cerámico en la ciudad de San José Cúcuta (Colombia) en el periodo 2008-2011".

** Estudiante del Doctorado en Estudios Políticos de la Universidad Externado de Colombia (Bogotá, Colombia), Magister en Administración de Empresas de la Universidad Nacional Experimental del Táchira (San Cristóbal, Venezuela), Especialista en Control Interno e Indicadores de Gestión de la Universidad de Pamplona (Colombia), Economista de la Universidad Libre (Bogotá, Colombia). Profesora asistente de tiempo completo, Directora del Departamento de Estudios Internacionales y de Fronteras y del Programa Comercio Internacional e integrante del grupo de investigación GIDSE en la Facultad de Ciencias Empresariales de la Universidad Francisco de Paula Santander (Cúcuta, Colombia). Correo electrónico: johannamogrovejo@ufps.edu.co

*** Magister en Administración de Empresas de la Universidad Nacional Experimental del Táchira (San Cristóbal, Venezuela), Especialista en Finanzas de la Universidad Autónoma de Bucaramanga (Colombia) y de la Universidad Francisco de Paula Santander (Cúcuta, Colombia), Economista de la Universidad Industrial de Santander (uis, Bucaramanga, Colombia). Profesora de tiempo completo e integrante del grupo de investigación GIDSE en la Facultad de Ciencias Empresariales de la Universidad Francisco de Paula Santander (Cúcuta, Colombia). Correo electrónico: lillianamarcelabo@ufps.edu.co

***** Magister en Administración de Empresas de la Universidad Nacional Experimental del Táchira (San Cristóbal, Venezuela), Administrador de Empresas de la Universidad Francisco de Paula Santander (Cúcuta, Colombia). Profesor de tiempo completo en la Facultad de Ciencias Empresariales de la Universidad Francisco de Paula Santander (Cúcuta, Colombia). Correo electrónico: jhonantunypabon@ufps.edu.co
} 
Fecha de recibido: 4 de septiembre de 2014

Fecha de aprobado: 17 de febrero de 2015

Doi: dx.doi.org/10.12804/rev.univ.empresa.29.2015.07

Para citar este artículo: Mogrovejo Andrade, J. M., Bastos Osorio, L. M., \& Antuny Pabón, J. (2015). Impacto económico del sector cerámico en San José de Cúcuta (Colombia). Universidad \& Empresa, 17(29), 157-180. Doi: dx.doi.org/10.12804/rev.univ.empresa.29.2015.07

\section{RESUMEN}

Esta investigación tuvo como finalidad determinar el impacto económico de las empresas del sector cerámico en la ciudad de San José Cúcuta (Colombia) en el periodo 2008-2011. Se utilizó como instrumento un cuestionario estructurado aplicado a los empresarios del sector. Igualmente, se efectuaron entrevistas a directivos de organizaciones gremiales. El impacto económico se analizó siguiendo la metodología propuesta por Soto y Bergoeing (1998), Arbeláez y Sandoval (2006) y Molina, Coronado y Rivera (2008). Se concluye que este sector, en el periodo estudiado, presentó gran impacto económico, en particular en lo relativo al nivel de empleo, el aporte en impuestos al municipio, el consumo intermedio y el valor agregado.

Palabras clave: empleo, impacto económico, producción, sector cerámico, valor agregado.

\section{ABSTRACT}

The purpose of this research was to establish the economic impact of the companies in the ceramic industry in the city of San José de Cúcuta (Colombia) in the period 2008-2011. A structured questionnaire was used as an instrument and applied to the sector. Executives of sectorial organizations were also interviewed. The economic impact was analyzed according to the methodology proposed by Soto and Bergoeing (1998), Arbelaez and Sandoval (2006) and Molina, Coronado and Rivera (2008). It was concluded that there had been considerable economic impact in this sector in the analyzed period, especially in the employment level, the contribution of municipal taxes, the intermediate consumption, and the added value.

Keywords: Employment, economic impact, production, ceramic industry, Added value.

\section{RESUMO}

Esta pesquisa teve como finalidade determinar o impacto económico das empresas do setor cerâmico na cidade de San José Cúcuta (Colômbia) no período 2008-2011. Utilizou-se como instrumento um questionário estruturado aplicado aos empresários do setor. Igualmente, efetuaram-se entrevistas a diretivos de organizações gremiais. O impacto económico se analisou seguindo a metodologia proposta por Soto e Bergoing (1998), Arbelaez e Sandoval (2006) e Molina, Coronado e Rivera (2008). Conclui-se que este setor, no período estudado, apresentou grande impacto económico, em particular, no relativo ao nível de emprego, o aporte em impostos ao município, o consumo intermédio e o valor agregado.

Palavras-chave: emprego, impacto económico, produção, setor cerâmico, valor agregado. 


\section{INTRODUCCIÓN}

En el campo económico y administrativo resulta importante el estudio de las micro, pequeñas y medianas empresas (mipymes). Ello teniendo en cuenta que son multiplicadoras de empleo en todos los niveles de la estructura productiva, ellas ocupan un lugar importante en este aspecto desde la contratación de mano de obra no calificada hasta los niveles técnicos. De allí que tengan una amplia capacidad de absorción y una constante oportunidad para integrarse verticalmente con otras industrias.

Este trabajo se orienta a un sector específico que tradicionalmente ha caracterizado al municipio de San José de Cúcuta (Colombia) y su zona de influencia, como es la explotación de la arcilla y la comercialización de sus derivados. Se busca con él, en concreto, determinar el impacto económico del sector cerámico en este municipio, entender su entorno y describir el comportamiento de factores y variables económicas que impactan en el mismo.

El desarrollo de los países de América Latina, en general, y de Colombia y sus regiones, en particular, requiere un proceso de cambio en el que los empresarios de las mipymes ocupan un lugar cada vez más destacado y determinante. Esto en tanto agentes activos en la generación de riqueza, empleo y bienestar social, así como en el restablecimiento del equilibrio económico. Estos aspectos constituyen una parte integral de los planes de desarrollo económico y social del Estado, que se implementan a través de programas de asistencia para el mejoramiento de la competitividad de esas estructuras.

Ilustrando de algún modo lo anterior, y las realidades contemporáneas, Stiglitz (2003), refiriéndose a la denominada 'nueva economía', señaló lo siguiente:
Hacia el final del decenio lo que se había saludado como el alba de una nueva era empezó a parecerse cada vez más a esas ráfagas de actividad, o hiperactividad, económica que in- variablemente desembocan en una crisis, obedeciendo a una ley que venía caracterizando al capitalismo durante doscientos años. Solo que esta vez la burbuja, el auge de la economía y el alza de la bolsa eran más importantes, como también lo serían sus consecuencias; y la nue- va era empezaba también para el resto del mundo, no solo para Es- tados Unidos. Como consecuencia, la crisis que siguió no solo afectaría a Estados Unidos, sino que se hizo sentir por todo el planeta. Eso no era lo previsto. (p. 37).

La situación descrita por este autor afectó a economías como las de los 
países en vía de desarrollo y afectó, entre otras, a la industria colombiana. Ante esta situación, el Estado colombiano promulgó la Ley de Fomento a las mipymes. Esta incluyó modificaciones a la prelación de las acreencias y estímulos a la banca cuando esta capitalice empresas, lo mismo que la recalificación de la cartera de compañías en concordato. Se formuló como una estrategia central, el fortalecimiento del Fondo Nacional de Garantías. Ello para respaldar las reprogramaciones y los nuevos créditos. Este hecho condujo a impulsarlas y a generar más empleosbasados en estetipode empresa.

En el departamento de Norte de Santander las pymes industriales representan cerca del $29 \%$ de la producción y del $42 \%$ del empleo del sector, lo que demuestra la importancia del sector para la economía local. Por esta razón, es importante fortalecer las mipymes de la industria de la cerámica, mediante la preservación de una cultura regional y el desarrollo económico local, a fin de lograr en forma duradera una presencia de esta en la economía mundial, es decir, en el interior de un proceso globalización. Autores como Pinto (2004) consideran que:

La trama y la urdimbre del tejido social están hechas de municipios, corregimientos y veredas. En forma equivocada algunos han llegado a pensar que el desarrollo local riñe con la decisión de lograr para la economía de nuestros países una sólida inserción en la economía mundial (p. 1).

El fortalecimiento del tejido empresarial puede vigorizar, en efecto, de acuerdo con lo señalado, la situación financiera de las organizaciones locales, del propio municipio y de su comunidad en general.

En el período de estudio de esta investigación, la caída vivida por el sector industrial colombiano se reflejó también en la ciudad de San José de Cúcuta. Se vio afectada, en particular, por los cambios ocurridos en Venezuela, en especial por la devaluación del bolívar frente al peso colombiano. En este contexto se presentó un decrecimiento en la dinámica empresarial, corroborado por informes de la Cámara de Comercio de Cúcuta, en donde se determinó que para el año 2001 existían 11453 empresas registradas, las cuales aumentaron a 11955 en el año 2002, pero que en el año 2003 mostraron una caída, apareciendo registradas solo 7449 empresas, de las cuales 89 corresponden al sector de minas y 940 , a la industria manufacturera.

La disminución de las unidades productivas ocasionó un deterioro 
en la estructura empresarial local. Esto obligó a muchas mipymes a suspender actividades, lo que originó un desempleo que superó la tasa histórica de la ciudad, lo que implicó una serie de consecuencias negativas en el sector productivo de San José de Cúcuta, en especial en la industria de la cerámica. A esta situación desfavorable, además, se sumaron los limitados resultados en la actividad edificadora local.

Considerando lo anterior, surgió el interés por conocer el impacto económico del sector de la cerámica en San José de Cúcuta. Dentro de ese marco se planteó como objetivo de la investigación determinar el impacto que en esta materia tienen las empresas del sector cerámico en la ciudad en el periodo 2008-2011.

El presente artículo está estructurado de acuerdo con el siguiente orden: en primer lugar se presenta la revisión de la literatura, en esta se presentan los elementos esenciales del marco teórico que, a su vez, permite la identificación del marco conceptual útil al análisis del impacto económico. En esta sección se considera una serie de estudios relacionados con el tema en el ámbito nacional e internacional. Posteriormente se presenta la metodología del trabajo. Luego se exponen los resultados del estudio y se hace la discusión de los mismos y, por último, se sintetizan las principales conclusiones de la investigación.

\section{REVISIÓN DE LA LITERATURA}

En el presente trabajo se consideran las siguientes definiciones básicas:

- Población económicamente activa: Supone todo trabajo por el cual se obtiene o genera un ingreso. Por lo tanto, no están solo aquí incluidos los trabajadores que reciben un salario, sino también aquellos que laboran de manera autónoma, es decir, toda la población que se encuentran ocupada. Esto en contraposición de aquella que se encuentra desocupada.

- Impacto económico: El conjunto de efectos sobre la producción, la renta y el empleo que tienen su origen en las variaciones de la demanda final de la producción de bienes y servicios.

- Impactos económicos directos: vienen recogidos por los incrementos en la demanda final, es decir, los gastos e inversiones públicas en los distintos sectores productivos.

- Impactos económicos inducidos: Son aquellos efectos que se generan en el entorno o región como consecuencia del efecto multiplicador sobre la economía de los impactos directos. 
- Producción: Se denomina producción a cualquier tipo de actividad destinada a la fabricación, elaboración u obtención de bienes y servicios.

- Renta: Comprende el incremento neto de riqueza. Todo ingreso que una persona física o jurídica percibe y que puede consumir sin disminuir su patrimonio.

En relación con las investigaciones enfocadas en el desarrollo de las mipymes, se presentan antecedentes que muestran la homogeneidad de la industria y las características del sector, investigaciones realizadas en el municipio San José de Cúcuta (Colombia) y en el estado Táchira (Venezuela). Estos son aportes importantes, por cuanto dichas regiones fronterizas tienen algunas similitudes y la estructura industrial y el flujo comercial es sui generis respecto a otras áreas geográficas del interior de los dos países.

De acuerdo con Bautista (2005), la apertura económica ha servido para mejorar el intercambio comercial entre Venezuela y Colombia. Sin embargo, las exportaciones del sector cerámico han experimentado al- tibajos. Ello principalmente debido a las drásticas fluctuaciones en las economías de ambos países, las cuales son fuertemente influenciadas por las devaluaciones y los controles de cambio en Venezuela. Para Becerra, Ramírez y Gelvez (2002), lo que se observa es la falta de diversificación de productos, el contrabando que se ha disparado desde este país y el incremento de los costos para los constructores.

Castro (2002) destaca que la arcilla nortesantandereana tiene un alto reconocimiento a nivel mundial. Este autor identificó estrategias que han hecho posible, en función de esa característica, y de otras, que las organizaciones del sector se mantengan en funcionamiento. Ello a pesar de los problemas fronterizos influidos por la relación cambiaria como resultante de las devaluaciones del bolívar y otros factores relacionados.

Colmenares y Yánez (2004) determinaron que el departamento de Norte de Santander ocupa una posición de liderazgo en el comercio internacional de productos derivados de la arcilla ( (res $\left.^{1}\right)$ y que utiliza un $74 \%$ de su capacidad instalada. Es-

1 "Gres (palabra proveniente del francés grès, arenisca) es el término genérico que designa una pasta cerámica, formada por arcillas naturales, o mucho más común por una combinación de arcillas plásticas refractarias, materiales desgrasante añadidos, como sílice y siendo el fundente usado más común el feldespato. Sus principales características son su dureza, ser casi impermeable una vez cocido a su temperatura de sinterización vítrea. El rango de cocción oscila desde los $1200^{\circ} \mathrm{C}$ a $\operatorname{los} 1300^{\circ} \mathrm{C}$, dependiendo de su composición química" Ver https://es.wikipedia.org/wiki/Gres 
tos investigadores plantean además el mapa de la cadena productiva y los actores que intervienen en esta. Identifican asimismo la existencia de deficiencias para la formación de la cadena productiva de la cerámica, en particular, debido a la falta de una cultura de asociatividad.

Ahora bien, las teorías que sustentan la presente investigación están relacionadas con los principios del pensamiento económico. Este abarca, en un contexto amplio, las intenciones de los inversionistas para desarrollar negocios y, dentro de esa nomenclatura, se entiende toda actividad económica, el sector manufacturero concretamente, buscando la analogía que existe en las industrias procesadoras de la arcilla. Las bases conceptuales gravitan alrededor de las teorías económicas relativas a las ventajas comparativas y competitivas, la rentabilidad, la productividad, la generación de empleo y el libre mercado, así como la prospectiva, la política cambiaria colombiana, las estrategias organizacionales y los aspectos relacionados con la cerámica propiamente dicha.

En relación con las teorías económicas y las variables que influyen en la gestión de las mipymes, se ha explicado desde hace muchos años que la labor empresarial está vinculada con una serie de actividades moti- vadas por los intereses del hombre en la generación de bienestar para quien desempeña una actividad económica y asume los riesgos. Según Case y Fair (1997) "la empresa como unidad de producción constituye una afirmación o conjunto de afirmaciones conexas sobre causa y efecto, acción y reacción" (p. 11). De acuerdo con estos autores, se trata entonces de entender la relación de causa y efecto que prevalece en una actividad económica.

El sector de la cerámica, al igual que otras industrias colombianas, busca obtener dividendos por sus actividades económicas, para lograrlo debe afrontar los nuevos retos, propios de la globalización, y una competencia directa con otros actores en materia de calidad, productividad, costos y precios. Habida cuenta de que los procesos de industrialización y el éxito de muchas empresas, estos elementos están asociados de diversos modos a las condiciones presentes en la región en la que se encuentran las compañías. Esto tiene que ver con variables como el ambiente de inversiones. No en vano los inversionistas extranjeros establecen una serie de parámetros para ubicar su capital en economías emergentes, en espera de obtener el esperado retorno sobre el capital.

Sobre este aspecto Aliber (1999) indica que las inversiones directas 
reflejan el hecho de que los países son receptores de inversión. Esto cuando existen niveles de rentabilidad marginal superiores a la rentabilidad del inversionista en su propio país de origen. Las diferentes tasas de capitalización en las inversiones extranjeras se producen cuando los niveles de tasa de rendimiento en el propio país tienden a ser superiores a las presentes en el exterior, es entonces cuando este se convierte en un mercado llamativo. Lodge (1996), considerando lo anterior, sugiere que los empresarios latinoamericanos exigían una rentabilidad de sus inversiones superior a las exigidas por los inversionistas extranjeros. Esto explicaría por qué en no pocos casos las inversiones nacionales pasan a manos de empresas extranjeras.

Existen entonces diferentes factores a considerar en el estudio, como los de actividad, endeudamiento y rentabilidad, los cuales, de acuerdo con Ortiz (1998), constituyen la forma más común de análisis financiero. Según este autor, "los indicadores de rentabilidad sirven para medir la efectividad de la administración de la empresa para controlar los costos y gastos de esta manera, convertir las ventas en utilidades" (p. 143). Estos aspectos, de cualquier modo, son elementos relevantes a estudiar dentro de las empresas. Es necesario evaluar cómo estos afectan su funcionamiento e impactan la economía local.

En relación con los planteamientos disponibles en materia de ventajas comparativas y competitivas, se encuentra que autores como Seldon \& Pennance (1990) han estudiado la presencia de ventajas superiores para la elaboración y comercialización de productos de una nación frente a otra. La competitividad, de acuerdo con ellos, se debe, entre otros factores, a una minimización de los costos y, por lo tanto, la eventual reducción de los precios finales del producto, los cuales, de este modo, pueden resultar ser inferiores $\mathrm{y}$ ventajosos para ser vendidos en otra región. De acuerdo con Porter (1990), la competitividad se relaciona con la posibilidad de desarrollar y conservar ventajas competitivas, de ser identificable al interior de un mercado y de dar valor agregado al producto. Esta surge por la incorporación de una serie de atributos $\mathrm{y}$ avances tecnológicos que hacen diferente la calidad y le incorporan al producto características nuevas, así como una mayor utilidad y exclusividad. En ese momento se logra una mayor competitividad. Con base en lo anterior, las empresas no solamente pueden considerar como fundamento de su competencia factores como la existencia de materia prima y el control sobre la misma, 
deben evaluar además otros aspectos que son también esenciales.

La ciencia económica contribuye de manera destacada su conocimiento al desarrollo socioeconómico. Esto, por ejemplo, a través de la microeconomía, la cual aporta una serie de principios para regir las unidades productivas, y de la macroeconomía, que contiene todas las variables que forman la interpretación global de lo que sucede a nivel de la producción. Según Case y Fair (1997) la macroeconomía es la rama "que estudia el comportamiento económico de los agregados ingreso, empleo, producción, inflación y así sucesivamente a nivel nacional" (p. 8). Este dominio teórico provee una mirada fundamental para el análisis, ya que la rentabilidad y el empleo que genera el sector de la cerámica se ven reflejados en la economía regional y nacional. De acuerdo con estos mismos autores, la microeconomía, por su parte, estudia el "funcionamiento de las industrias individuales, tomadoras de decisiones es decir, las empresas comerciales y las familias" (p. 8), lo cual permite también, en materia de análisis, tener una más clara expresión del comportamiento de variables macroeconómicas que impactan en la eficiencia de una empresa.

El empleo, por su parte, constituye una de las mayores preocupaciones de los Gobiernos. La estabilidad social depende fundamentalmente de esta variable. Las empresas, además de obtener utilidades en sus respectivos contextos de acción, contribuyen a atenuar los problemas sociales presentes en estos mediante la generación de empleo para la masa trabajadora y, a través de ello, los ingresos familiares que contribuyen a su supervivencia y bienestar. Este tema, por lo que implica, es tratado desde Keynes (1944 citado en Maza, 2000) con el ideal de llegar a alcanzar el pleno empleo. El Estado, para aproximarse a ese ideal, se ve en la necesidad de impulsar la creación de puestos de trabajo, entre otras acciones, a través de actividades de inversión pública.

Las empresas para ser competitivas y proveer empleo en sus regiones, aportando con ello al desarrollo social y al cumplimiento de las metas del Estado, deben enfrentarse a un mercado que, en buena medida, se regula bajo el sistema de precios. Este, de acuerdo con Friedman y Friedman (1996):

Es el mecanismo mediante el cual dos o más individuos se pueden entender aun sin conocerse e independientemente cuan distantes se encuentre uno del otro, sin necesidad de una dirección centralizada, sin obligar a la personas a hablar entre sí, o que se entiendan mutuamente (p. 30). 
Esta reflexión respecto al mercado sigue vigente, en particular, considerando que las operaciones comerciales y financieras en la actualidad se realizan desde muy diferentes $-y$ en no pocos casos- distantes lugares. Esto, haciendo uso de las tecnologías de la información y las comunicaciones (TIC). En la actualidad, como resultado de la llegada y el auge de estas tecnologías, el mercado es un lugar altamente digitalizado en donde cada empresa trata de ubicar su producto y venderlo compitiendo con una gran cantidad y variedad de productos similares.

La arcilla es un mineral que requiere, por su naturaleza, importantes procesos de transformación en hornos de altas temperaturas. Los procesos de producción vinculados con ella son generadores de empleo y este último, como ya se ha indicado, genera un impacto social positivo que, cuando no se hace presente, plantea destacadas dificultades para las regiones. Según Londoño (2001), en efecto, "la incapacidad del aparato productivo para generar empleos crea problemas adicionales de tipo social. El proceso inflacionario que se ha vivido en las últimas décadas y el deterioro el valor de los salarios" (p. 45). Sin embargo, generar empleo, así como ser competitivo y perdurar, constituye una labor de gran complejidad. Ello por la can- tidad de factores que lleva implícito su desarrollo, los cuales no dependen solo de la voluntad y los buenos deseos del empresariado, sino de un conjunto de condiciones, como las mencionadas arriba, que contribuyen o no a impulsar las iniciativas particulares generadas en esta materia.

De acuerdo con Domínguez (2001), las pequeñas y medianas empresas (pymes), en este contexto, ocupan un lugar fundamental para mover a la economía. Sin embargo, el esfuerzo por generar negocios es limitado debido, entre otras razones, a la falta del conocimiento requerido por los empresarios. De hecho, la principal razón de la importancia que tienen las mipymes en la economía es su capacidad para generar empleo e impactar en otros sistemas externos a ella. Pero, para poder hacer esto de manera adecuada, requiere generar las sinergias internas necesarias, comprendiendo que en su interior, cada variable cuenta al impactar sobre las demás. Porter (1990), en ese sentido, plantea que efectivamente

La actividad de cualquier parte de una organización afecta la competitividad de cualquier otra [...] entonces, en los sistemas no hay unidades aisladas, por el contrario todas sus partes actúan con una misma orientación y satisfacen un objetivo común [...] es necesario el funciona- 
miento correcto de las partes para el eficaz desempeño del todo en su conjunto (p. 27).

Para poder impactar de manera adecuada en el medio y competir en el mercado, es necesario interactuar de forma sinérgica con otras organizaciones. Esto implica considerar aspectos como la cadena de valor e identificar el eslabonamiento más conveniente de cada unidad productiva y las posibilidades de integración horizontal y vertical que esta posee. Este tipo de integración es esencial, en lo fundamental, para mipymes como las presentes en industrias como la de la cerámica y en lugares como la ciudad de San José de Cúcuta. De acuerdo con Rego (2003), en particular

La integración hacia adelante es, al parecer la fórmula para romper el paradigma y mejorar la posición competitiva de las empresas basadas en un acercamiento al consumidor final, un manejo racional de recursos a lo largo de la cadena que conforma la producción, la consolidación significativa de los canales de distribución, la retroalimentación directa con el mercado y la minimización de los riesgos; [estas] son algunas de las ventajas que la integración hacia delante proporciona en la búsqueda de competitividad (p. 19).

Así, la integración del sector cerámico es, según los elementos teóricos mencionados, una importante fórmula para mejorar a nivel empresarial y agregar valor a las organizaciones para que estas puedan ser competitivas en su industria a nivel nacional e internacional.

El desempeño empresarial no es una actividad económica aislada. En todo proceso que se relaciona con la producción se toman en cuenta factores tanto internos como externos (entre ellos el rol del Estado, como ente promotor, y de las autoridades locales). Es necesario considerar la serie de aspectos que pueden llegar a contribuir al buen desempeño económico de las empresas y de sus regiones, puesto que ambos están en permanente relación de interacción y de mutua dependencia. El éxito de las empresas es vital para el desarrollo del municipio, de la región y del país, pero, a su vez, para que este pueda darse es necesaria la existencia de oportunidades en el entorno, así como de facilidades para el desarrollo de iniciativas empresariales y la presencia de un ambiente jurídico y económico estable.

En el caso de las industrias del municipio de San José de Cúcuta, se presenta una situación particular y positiva para el desarrollo de las empresas procesadoras de arcilla, en particular, por la existencia allí de una abundante materia prima, lo que 
constituye una gran oportunidad para el aprovechamiento de este recurso en la generación de mejores condiciones socioeconómicas, a través del incremento en los niveles de la productividad y la competitividad por la vía, por ejemplo, de la innovación. Al respecto conviene considerar los aportes de Shumpeter (1976), quien se refirió a esta como un principio económico que contribuye a lograr cambios destacados en el desarrollo y elaboración de productos y a mejorar la competitividad. Ello a través de la implementación de mecanismos entre los que se destaca el de la "destrucción creativa". Sus aportes siguen siendo vigentes y de ayuda para visualizar el necesario cambio en la visión del empresario moderno, quien tiene el reto de enfrentar mercados altamente desarrollados y dinámicos.

Así, existe una gran cantidad de efectos positivos alrededor de la realización de proyectos de producción específicos, vinculados en particular con las mipymes, no solo para las propias empresas y sus empresarios, sino para el bienestar y el desarrollo de los municipios, las regiones, los países en los cuales estos se desarrollan (Centro de Investigaciones para el Desarrollo, Universidad Nacional de Colombia, 1994). De su productividad, competitividad y capacidad de innovación dependen no solo sus posibilidades de supervivencia y crecimiento, a nivel endógeno, sino también un sinnúmero de alcances esenciales en su entorno, los cuales impulsan el crecimiento socioeconómico. Estos elementos teóricos son considerados como esenciales para la presente investigación.

\section{METODOLOGÍA}

La investigación se acometió bajo un enfoque cuantitativo, complementado con elementos del análisis cualitativo. Esto en virtud de la consulta que se efectuó a personas relacionadas con el sector en estudio (Quivy \& Van Campenhoudt, 2006). Respecto al nivel o tipo de investigación, esta se desarrolló como descriptiva-correlacional. El diseño de la investigación fue de tipo no experimental, documental y de campo. El trabajo documental se efectuó mediante la revisión de información, considerando que el sector puede ser diagnosticado y medido en la realidad estudiada, tal y como esta se presenta. En el estudio de campo se aplicó un cuestionario de preguntas cerradas a personas relacionadas directamente con las mipymes del sector de la arcilla y cerámico de San José de Cúcuta.

La población objetivo de la investigación está conformada por veinte fabricantes o tejares de productos 
derivados de la arcilla de San José de Cúcuta. Dado que se trata de conocer los aspectos fundamentales de las mipymes productoras de los derivados de la arcilla, se realizó un censo, correspondiente a aquellas industrias consolidadas, por cuanto la población es pequeña.

En cuanto a la recolección de datos, se utilizó como instrumento un cuestionario estructurado aplicado a los empresarios del sector y se entrevistó a directivos de organizaciones gremiales. Para certificar la pertinencia, relación y validez interna del contenido, se utilizó la técnica de juicio de expertos. El modelo de confiabilidad en el cuestionario se basó en el alfa de Cronbach, y se obtuvo como resultado 0,80. Este valor, de acuerdo con lo estimado usualmente en la literatura especializada, se considera alto.

Por su parte, el impacto económico se analizó mediante la metodología propuesta por Arbeláez y Sandoval (2006), Molina, Coronado y Rivera (2008) y Soto y Bergoeing (1998). Siguiendo esa metodología, se efectuó una aproximación del impacto directo y del impacto indirecto del sector cerámico el cual, según el Departamento Administrativo Nacional de Estadística-DANE- (2007) de Colombia, aparece en la Clasificación Internacional Industrial Unifor- me (CIIU), revisión 3, de la siguiente forma:

- División 26 Fabricación de otros productos minerales no metálicos.

- Grupo 269 Fabricación de productos minerales no metálicos NCP.

- Clase 2693 Fabricación de productos de arcilla y cerámica no refractaria, para uso estructural.

En el estudio de la información documental se aplicó análisis matemático y se utilizó la estadística descriptiva e inferencial. Finalmente, para el análisis de las encuestas, se aplicaron estadística descriptiva y distribuciones de frecuencia simple.

\section{RESULTADOS}

\subsection{Impacto económico}

Se determinó el impacto económico directo, considerando los empleos, sueldos e impuestos que se generan debido a la actividad económica. En primer lugar se definió el área de influencia del grupo económico. De acuerdo con información de la Cámara de Comercio de Cúcuta - $\mathrm{CCC}-$ (2012), el clúster de cerámica de Norte de Santander cuenta con sesenta y siete empresas productoras, las cuales están dedicadas a elaborar y comercializar productos derivados 
de la arcilla como bloques, tejas, tableta vitrificada, enchapes, decorados y rosetones, entre otros.

Se encontró que las empresas que conforman el sector son pequeñas y medianas, porque cuentan en promedio con un personal de sesenta empleados. Así mismo, se halló que la mano de obra es muy característica, representando en el costo del producto aproximadamente el $30 \%$. Se verificó que en los años 2006 a 2009 el aumento constante de la demanda dio lugar a que una serie de empresas se ubicaran en la ciudad de Cúcuta, Villa del Rosario, Los Patios, El Zulia y San Cayetano, pero que, a raíz del conflicto presentado en 2009 , año en el que el gobierno venezolano cerró las relaciones comerciales con Colombia, se generaron consecuencias negativas que dejaron un centenar de fabricantes de la arcilla sin el reintegro de divisas y llevándolas a declararse en quiebra. Esta situación dejó a muchas personas sin empleo.

Como se dice más arriba, de acuerdo con la información de la CCC, el clúster de la arcilla se encuentra conformado por sesenta y siete empresas fabricantes, las que en su mayoría son de carácter familiar o unipersonal. En la cadena de valor se identifica un número y variedad de actores destacados como constructores (210), ferreteros (722), ingenieros (72), arquitectos (28), maestros (54), empresas de empaque y embalaje (33), compañías de transporte de carga (234), empresas de aditivos y lubricantes (32) y universidades (3). En la figura 1 se muestra la cadena de valor del sector cerámica. 
Figura 1. Cadena de valor del sector de la cerámica

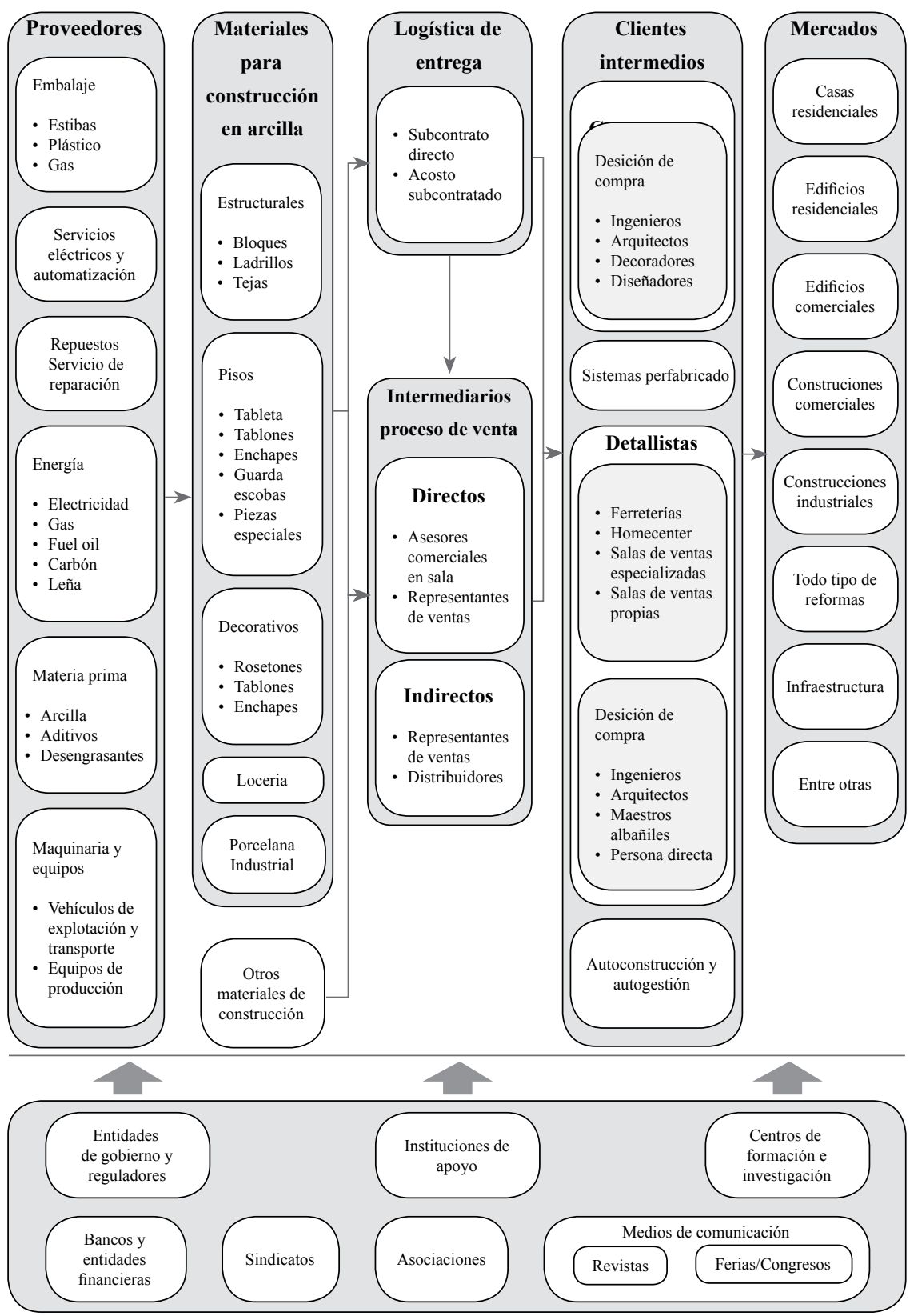

Fuente: Cámara de Comercio de Cúcuta (2012). 


\subsubsection{Generación de riqueza en la zona de influencia}

Con la información de la Encuesta Anual Manufacturera (EAM) del
DANE, de los años 2008 a 2011, se construyó la tabla 1 , en donde se presentan los impactos directos relacionados con los empleos, sueldos e impuestos.

Tabla 1. Impactos directos del grupo industrial 269 en la ciudad de Cúcuta

\begin{tabular}{|c|c|c|c|c|c|c|c|c|c|c|c|}
\hline Año & 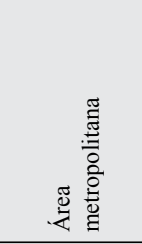 & 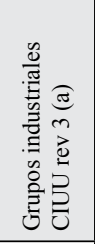 & 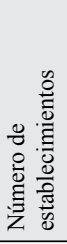 & 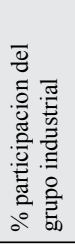 & 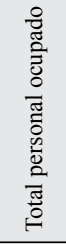 & 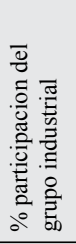 & 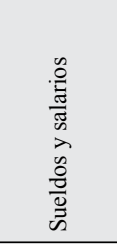 & 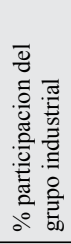 & 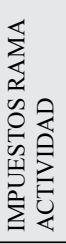 & 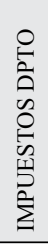 & 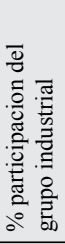 \\
\hline \multirow{2}{*}{2008} & Grupo Indust & 269 & 20 & $16,3 \%$ & 2.096 & $41,0 \%$ & 14.363 .814 & $42,0 \%$ & 160 & & $33 \%$ \\
\hline & Cúcuta & TOTAL & 123 & & 5.109 & & 34.167 .169 & & & 482 & \\
\hline \multirow{2}{*}{2009} & Grupo Indust & 269 & 24 & $17,6 \%$ & 2.123 & $43,1 \%$ & 14.565 .618 & $41,7 \%$ & 157 & & $33 \%$ \\
\hline & Cúcuta & TOTAL & 136 & & 4.925 & & 34.925 .910 & & & 471 & \\
\hline \multirow{2}{*}{2010} & Grupo Indust & 269 & 24 & $18,0 \%$ & 1.864 & $39,4 \%$ & 14.701 .380 & $41,2 \%$ & 30 & & $6 \%$ \\
\hline & Cúcuta & TOTAL & 133 & & 4.731 & & 35.711 .347 & & & 484 & \\
\hline \multirow{2}{*}{2011} & Grupo Indust & 269 & 25 & $18,2 \%$ & 1.903 & $38,1 \%$ & 14.862 .422 & $37,4 \%$ & 3 & & $1 \%$ \\
\hline & Cúcuta & TOTAL & 137 & & 4.993 & & 39.743 .649 & & & 591 & \\
\hline
\end{tabular}

Fuente: Elaboración propia a partir de cálculos realizados con datos de la EAM del DANE, años 2008 a 2011.

El número de establecimientos representó el 17,6\% de los instalados en la ciudad durante el periodo de estudio, con un promedio de personal ocupado de 1997 personas. Esto representó un promedio del $40,4 \%$. A su vez, los sueldos acumulados en el periodo, en términos corrientes, sumaron \$14623308,50 miles de millones de pesos, lo que significó un promedio de $40,6 \%$ de los sueldos en la ciudad.

En cuanto a los impuestos, se observó que su participación pasó del $33 \%$ en 2008 al $6 \%$ en 2010 y cayó al $1 \%$ en 2011 . Esta caída significativa en la participación de impuestos apuntala lo comentado sobre el impacto de la crisis que se presentó a raíz del conflicto fronterizo con Venezuela en 2008. Esto también se reflejó en la caída del porcentaje de participación del personal ocupado, como se observa en la figura 2.

La generación de riqueza en la zona de influencia se aproximó mediante los flujos de dinero que la actividad transfirió. Esto se relacionó con la producción bruta, el consumo intermedio y el valor agregado. Se de- 
terminó que la producción bruta acumulada alcanzó los \$1380465607 miles de millones de pesos, lo que representó en promedio el 39,9\% de la producción bruta de la ciudad en el periodo estudiado. El consumo intermedio acumulado fue de $\$ 520302471$ miles de millones de pesos, correspondiente al $27,9 \%$ del de la ciudad (ver tabla 2).

Figura 2. Porcentaje de participación de personal ocupado del grupo industrial 269

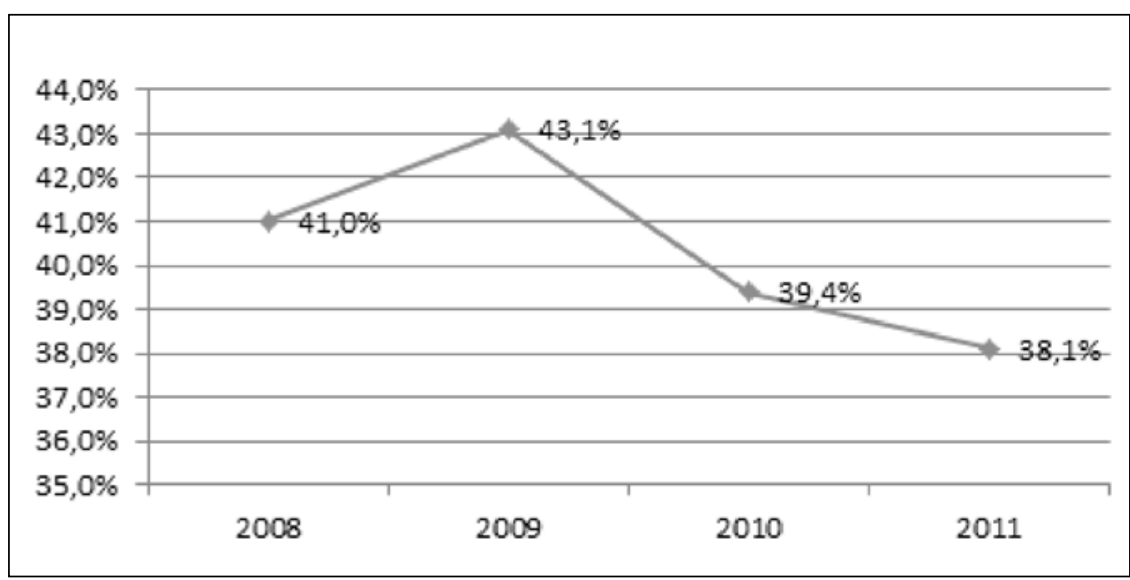

Fuente: Elaboración propia a partir de cálculos realizados con datos de la EAM del DANE, años 2008 a 2011.

Tabla 2. Generación de riqueza en la zona de influencia

\begin{tabular}{|c|c|c|c|c|c|c|c|c|c|c|c|c|}
\hline Año & 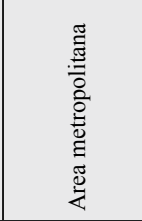 & 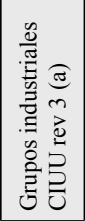 & 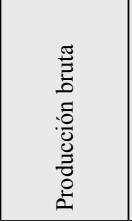 & 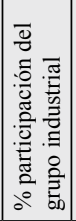 & 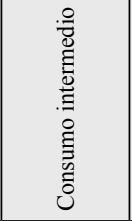 & 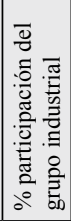 & 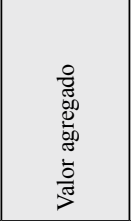 & 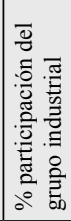 & 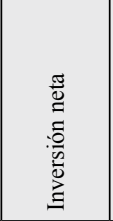 & 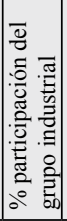 & 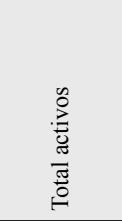 & 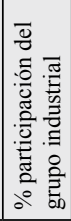 \\
\hline \multirow{2}{*}{2008} & Grupo Indust & 269 & 277.585 .848 & $38,6 \%$ & 101.615.308 & $26,9 \%$ & 175.970 .540 & $51,5 \%$ & -3.845 .944 & & 154.155 .708 & $49,4 \%$ \\
\hline & Cúcuta & TOTAL & 718.760 .743 & & 377.370 .939 & & 341.389 .804 & & 1.047 .463 & & 312.078 .095 & \\
\hline \multirow{2}{*}{2009} & Grupo Indust & 269 & 273.379 .088 & $41,2 \%$ & 106.814 .363 & $30,5 \%$ & 166.564 .725 & $53,1 \%$ & -8.382 .202 & & 160.604 .293 & $48,0 \%$ \\
\hline & Cúcuta & TOTAL & 663.512 .067 & & 349.778 .454 & & 313.733 .613 & & -801.441 & & 334.692 .512 & \\
\hline \multirow{2}{*}{2010} & Grupo Indust & 269 & 264.026 .348 & $41,2 \%$ & 92.516 .076 & $26,3 \%$ & 171.510 .272 & $59,2 \%$ & -4.795 .122 & & 141.159 .188 & $41,6 \%$ \\
\hline & Cúcuta & TOTAL & 641.522 .254 & & 351.640 .036 & & 289.882 .218 & & 5.237 .468 & & 339.387 .505 & \\
\hline \multirow{2}{*}{2011} & Grupo Indust & 269 & 287.888 .475 & $38,7 \%$ & 117.741 .416 & $27,7 \%$ & 170.147 .059 & $53,3 \%$ & & & 143.764 .008 & $41,4 \%$ \\
\hline & Cúcuta & TOTAL & 744.602 .410 & & 425.468 .919 & & 319.133 .491 & & & & 347.607 .191 & \\
\hline
\end{tabular}

Fuente: Elaboración propia a partir de cálculos elaborados con datos de la EAM del DANE, años 2008 a 2011. 
La participación del producto interno bruto (PIB) del sector cerámico en el PIB del Departamento de Norte de tra la figura 3, en donde se observa que este pasó del $8,6 \%$ en 2008 al Santander se comportó como mues$7,2 \%$ en 2011.

Figura 3. Porcentaje de participación del PIB del sector en el PIB del departamento

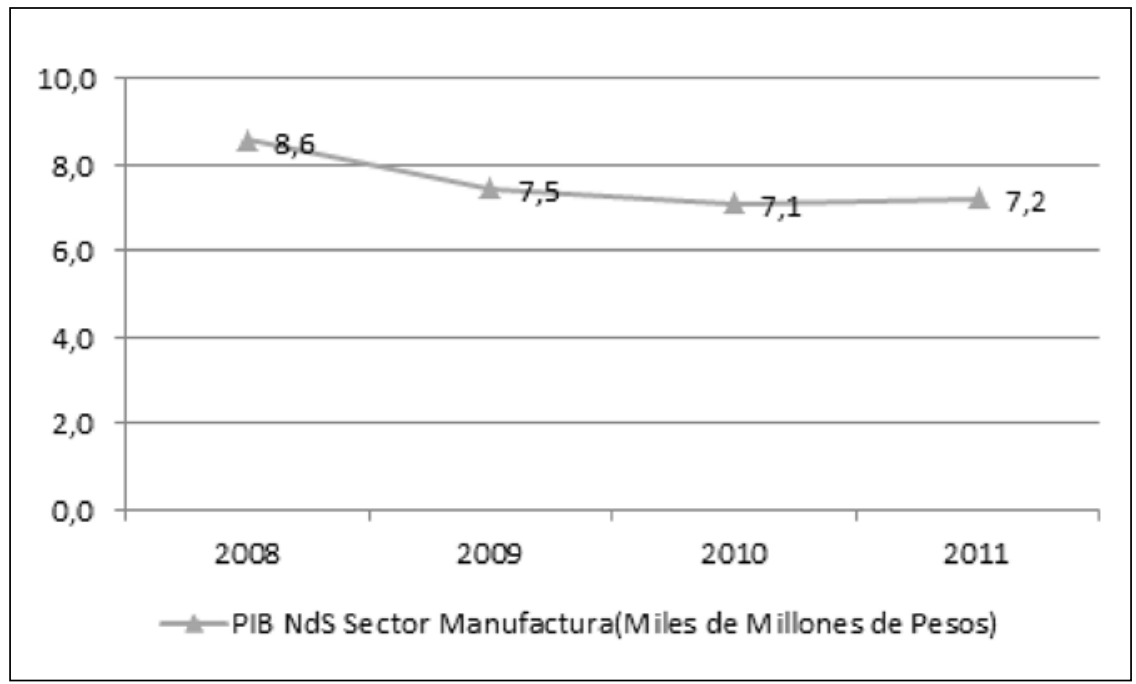

Fuente: Elaboración propia a partir de cálculos elaborados con datos de la EAM del DANE, años 2008 a 2011.

\subsubsection{Relaciones intersectoriales con la economía de la zona}

Al comparar el porcentaje del PIB del sector cerámico, considerando el promedio durante el periodo del presente estudio, con el de sectores relacionados como la explotación de minas y canteras, la industria manufacturera, la electricidad, el gas y el agua, la construcción, el comercio, la reparación, los restaurantes y hoteles, el transporte, el almacenamiento y las comunicaciones, los estable- cimientos financieros, los seguros, las actividades inmobiliarias, los servicios a las empresas y las actividades de servicios sociales, comunales y personales, se encontró que el sector con mayor porcentaje de participación correspondió al de energía (con el 47,3\%), seguido de los sectores de explotación de minas y canteras $(44,1 \%)$, construcción $(25,4 \%)$, industria manufacturera $(24,7 \%)$ y transporte y comunicaciones $(20,4 \%)$ (ver tabla 3$)$. 
Tabla 3. Relaciones intersectoriales con la economía de la zona

\begin{tabular}{|c|c|c|c|c|c|c|c|c|}
\hline $\begin{array}{c}\text { AÑO } \\
2008- \\
2011\end{array}$ & $\begin{array}{c}\text { Explotación } \\
\text { de minas y } \\
\text { canteras }\end{array}$ & $\begin{array}{c}\text { Industria } \\
\text { manufacturera }\end{array}$ & $\begin{array}{c}\text { Electricidad, } \\
\text { gas y agua }\end{array}$ & Construcción & $\begin{array}{c}\text { Comercio, } \\
\text { reparación, } \\
\text { restaurantes } \\
\text { y hoteles }\end{array}$ & $\begin{array}{c}\text { Transporte, } \\
\text { almacenamiento } \\
y \\
\text { comunicaciones }\end{array}$ & $\begin{array}{c}\text { Establecimientos } \\
\text { financieros, segu- } \\
\text { ros, actividades } \\
\text { inmobiliarias y } \\
\text { servicios a las } \\
\text { empresas }\end{array}$ & $\begin{array}{c}\text { Actividades } \\
\text { de servicios } \\
\text { sociales, } \\
\text { comunales y } \\
\text { personales }\end{array}$ \\
\hline $\begin{array}{c}\text { Prome- } \\
\text { dio }\end{array}$ & $44,1 \%$ & $24,7 \%$ & $47,3 \%$ & $25,4 \%$ & $14,9 \%$ & $20,4 \%$ & $12,7 \%$ & $8,2 \%$ \\
\hline
\end{tabular}

Fuente: Elaboración propia a partir de cálculos elaborados con datos de la EAM del DANE, años 2008 a 2011.

\subsubsection{Generación de empleo en la zona}

El empleo directo del sector cerámico alcanzó un promedio por año, en el período estudiado, de 1998 personas ocupadas directamente. Durante este espacio de tiempo el total de personas ocupadas fue de 7986 . De acuerdo con la metodología utilizada por Molina et al. (2008) se estimó que como empleo inducido o derivado de las operaciones se generaron 12880 puestos, de los cuales, tomando como promedio según cifras del DANE cuatro personas por núcleo familiar, se estimó que dependían 51521 personas.

\subsection{Resultados de la encuesta}

El análisis de la encuesta aplicada mostró que las mipymes indagadas generaron en el periodo de estudio cerca de 800 empleos directos y 2400 indirectos. Los técnicos de planta y administración no excedieron el $20 \%$ de personal capacitado profesionalmente en las empresas.
Entre los principales aportes económicos que ofreció el sector de las arcillas al municipio estuvo, justamente, según el $80 \%$ de los encuestados, la generación de empleos aunque también, de acuerdo con el $60 \%$ de los mismos, el pago de impuestos. $\mathrm{Al}$ analizar el aspecto administrativo de la mipymes de las arcillas, se encuentra que el $45 \%$ consideró que la mala administración fue el principal factor que afectó el capital de trabajo de la microempresa, esto aunado a la morosidad con proveedores.

Sobre el tema de los impuestos el $50 \%$ de los encuestados indicó que su pago es el aspecto financiero que más preocupa a los microempresarios del sector de las arcillas al considerarlo alto. En cuanto a la producción, el $75 \%$ de las empresas sobrepasó las 500 toneladas, entre estos el $40 \%$ produjo más de 1000 toneladas. Además, el $50 \%$ de las microempresas estaba produciendo entre un $60 \%$ y un $80 \%$ de su capacidad total. El $95 \%$ de los consultados consideró, asimismo, el año 
2006 como el de mejores resultados económicos. Esto debido al incremento de la exportación de los productos derivados de las arcillas al mercado venezolano.

Respecto a la apertura económica, el $50 \%$ de los individuos consultados consideró que esta permitió la creación de nuevas industrias de la arcilla, aunque también que la misma constituyó una amenaza para las empresas ya establecidas. El 40\% indicó que la apertura había sido positiva, ya que por ella, en particular, incrementaron las exportaciones hacia el mercado venezolano.

\section{DISCUSIÓN}

La mayoría de las empresas en el período estudiado no produjo a su máxima capacidad. Esto fue desventajoso desde el punto de vista financiero, ya que se debieron cubrir los costos fijos con la producción actual y, al no aumentar los niveles de producción, el costo fijo medio se incrementó, lo que corresponde con lo previsto en un principio económico básico de la economía de escala que, en adelante, debe considerarse para la planificación de las empresas. Ello en consonancia con lo que a nivel teórico plantean autores como Case y Fair (1997), Goodman (2000), Miller y Leroy (1988) y Samuelson y Northdaus (2002).
Esta situación restó capacidad competitiva a las empresas estudiadas. Dicho hallazgo se corresponde con lo presentado por Sánchez (2014) en su análisis acerca de la crisis vivida en la frontera en San José de Cúcuta, entre Colombia y Venezuela.

Al confrontar la caracterización del sector en estudio con los factores de competitividad, de acuerdo a lo planteado por autores como Porter (1990), se pudo determinar que existe una serie de debilidades que minimizan su potencialidad de inserción en mercados competitivos. Esta es una oportunidad que se presenta para que todos los sectores relacionados (gobierno, academia, industria y gremios) aporten en la propuesta estratégica necesaria para fortalecer al sector, de acuerdo con lo esbozado por investigadores como David (2002) y Serna (2000). Se corrobora con este trabajo, además, la importancia de utilizar la metodología de Molina et al. (2008) para determinar el impacto económico directo e indirecto sobre la economía del departamento de Norte de Santander, específicamente, de las mipymes de la industria de la arcilla en San José Cúcuta para el periodo 2008-2011.

Los hallazgos constituyen un aporte valioso para el conocimiento, con sustento empírico, de dicho sector. La utilización de sus resultados 
puede servir de apoyo al desarrollo e implementación de los planes que se generen para la región. Esto en considerando lo expuesto por autores como Arbeláez y Sandoval (2006), Molina et al. (2008) y Soto y Bergoeing (1998).

\section{CONCLUSIONES}

Partiendo, en lo fundamental, de los hallazgos del trabajo de campo y la información documental para determinar el impacto económico de las mipymes de la industria de la arcilla en San José Cúcuta en el periodo 2008-2011, se concluye lo siguiente:

En el periodo de estudio, el personal con nivel de capacitación profesional no alcanzó el $20 \%$ del total de empleados de la planta.

La explotación en la mina no se realizó con las técnicas más adecuadas. Esto ocasionó mezclas inapropiadas y generó problemas de erosión y de sedimentos con daños ambientales. No existió además asesoría técnica para optimizar el proceso desde su extracción en mina hasta su cargue. Este fenómeno se presentó con mayor fuerza en el sector artesanal, en donde el desarrollo tecnológico en este renglón económico fue muy bajo, de hecho, casi inexistente.

La mayor producción estuvo conformada por bloques, seguida de tejas y tabletas. Existieron otros productos que, aunque no eran los principales (enchapes, ladrillos y otros), representaron una cantidad significativa de materiales para la venta al sector de la construcción y fueron exportados hacia Venezuela y Norteamérica.

El sector de la arcilla ha sido muy importante, tanto para la captación de recurso humano del municipio como por el pago de impuestos al fisco local. La carencia de capital de trabajo ha conducido a que muchas de estas empresas sean tan solo de subsistencia y a que múltiples familias que trabajaban allí vivieran en condiciones precarias sin oportunidades de crecimiento económico y de mejoramiento de su bienestar.

El empleo fue un factor importante que afectó desde las empresas al municipio y su sociedad en general. El $60 \%$ de los informantes indicó que muchas veces debieron prescindir de trabajadores debido, entre otras causas, por ejemplo, al incremento de los costos en los servicios básicos, los recursos y los materiales. Esto, por supuesto, tuvo un impacto económico negativo en la ciudad.

La inexistencia de acueducto en algunos sectores incrementó también el costo del producto. Ello debido a la necesidad de adquirir agua me- 
diante carros tanque. La mayoría de chircales artesanales estaban ubicados sobre terrenos de invasión. En estos lugares las continuas extracciones de arcilla ocasionaron desestabilización en los terrenos e incrementaron el riesgo generado por la actividad.

La apertura económica fue el inicio de nuevos negocios internacionales y sus consecuencias fueron positivas para algunas empresas en la industria, aunque, como es natural, para para otras, no. Esto dado principalmente el hecho de que se quedaron rezagadas y no lograron ponerse a tono con las nuevas exigencias del mercado.

Se determinó que el sector cerámico tiene un peso importante en la economía de la ciudad de San José de Cúcuta en el departamento de Norte de Santander. Este es relevante en la generación de empleos y de riqueza para la región, ello considerando la participación porcentual del PIB del sector cerámico en la economía, lo que la constituye en uno de los principales sectores del municipio. Este hecho no solo se reflejó en los resultados de la encuesta, sino en los indicadores identificados a nivel nacional.

Se concluye, por último, que el sector, en el periodo estudiado, pre- sentó un alto impacto económico directo en relación con el nivel de empleo, el aporte en impuestos al municipio, el consumo intermedio y el valor agregado. Así mismo, se verificó el impacto inducido sobre la economía de la región debido a las operaciones del sector cerámico, lo que contribuye también al desarrollo de otros sectores.

\section{REFERENCIAS}

Arbeláez, M.,\& Sandoval,C. (2009). Contribución de la operación de Bavaria S.A en la economía colombiana. Mimeo. Fedesarrollo. Recuperado de http://www.fedesarrollo.org.co/wp-content/ uploads/2011/08/comunicados Fedesarrollo-Impacto-economico-de-Bavaria-Informe-definitivo.pdf

Aliber, R. (1999). Portafolio global. Caracas: $\mathrm{BCV}$.

Bautista, C. (2005). Incidencias de la apertura económica de las exportaciones de materiales cerámicos originarios de Cúcuta (Tesis inédita, Universidad Nacional Experimental del Táchira, San Cristóbal, Venezuela).

Becerra, C., \& Ramírez, L., \& Gelvez, J. (2002). Investigación de mercado para los productos de arcilla y sus derivados en Cúcuta y su área metropolitana (Tesis inédita, Universidad Francisco 
de Paula Santander, Cúcuta, Colombia).

Cámara de Comercio de Cúcuta(CCC) (2012). Documento del clúster de cerámica de Norte de Santander. Recuperado de http://media. wix.com/ugd/57f688_2a42bc08 64dc4eaf8341b2b94b1fcb5c.pdf

Case, K., \& Fair, R. (1997). Principios de microeconomía (4a ed.). México: Prentice-Hall Hispanoamericana.

Castro, A., \& Sayago, P. (2002). Análisis situacional y económico de las micropymes y la gran empresa de los sectores industriales de la arcilla (Tesis inédita, Universidad Francisco de Paula Santander, Cúcuta, Colombia).

Centro de Investigaciones para el Desarrollo(CID), UniversidadNacional de Colombia (1994). Programa de capacitación de asesoría para el departamento de Arauca. Recuperado de www.cid.unal. edu.co/files/publications/CID199411loprca.pdf

Colmenares, D., \& Yánez, J. (2004). Plan estratégico para el sector productivo derivados de la arcilla (gres), en el área metropolitana de Cúcuta orientado hacia el diseño de la cadena productiva (Tesis inédita, Universidad Francisco de Paula Santander, Cúcuta, Colombia).

David, F. (2002). Fundamentos de planificación estratégica. México: McGraw-Hill.
Departamento Administrativo Nacional de Estadística (DANE) (2007) Clasificación industrial internacional uniforme de todas las actividades económicas. Revisión 3.1 adaptada para Colombia CIIU Rev. 3.1 A.C. Recuperado de http://www.dane.gov.co/index. $\mathrm{php} /$ normas-y-estandares/nomenclaturas-y-clasificacionessen/160-uncategorised/2723clasificacion-industrial-internacional-uniforme-de-todas-lasactividades-economicas-ciiu

Departamento Administrativo Nacional deEstadística (DANE) (2008, 2009, 2010, 2011). Encuesta Anual Manufacturera. Recuperado de http://www.dane.gov.co/ index.php/industria/encuestaanual-manufacturera-eam

Domínguez, L. (2001). Pymes en Norte de Santander: ¿por qué no aprovechan las oportunidades? Recuperadodehttp:/www.gestiopolis.com

Friedman, M.,\&Friedman, R.(1996). La libertad de elegir. México: Grijalbo.

Goodman, S. (2000). Administración de efectivo para las pymes. México: McGraw-Hill.

Lodge, G. (1996). Estudio de actitud de empresas. Caracas: BVC.

Londoño, G. (2001). Estructura económica colombiana. Bogotá:McGraw-Hill.

Maza, D. (2000). Metodología de la macroeconomía. Caracas: Ávila. 
Miller, R. \& Leroy, M. (1988). Microeconomía. $3^{\text {a }}$ ed.). México: McGraw-Hill.

Molina, J., Coronado, C., \& Rivera, G. (2008). Aproximación al impacto económico local de la minería aurífera: el caso de Mineros S.A. Boletín de Ciencias de la Tierra. (24), p. 19-27.

Ortiz, H. (1998). Análisis financiero aplicado con ajustes por inflación. Bogotá: Universidad Externado de Colombia.

Pinto, J. (2004). Los alcaldes y las pymes. ElTiempo.com. Recu-

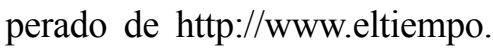
com/archivo/documento/MAM1523667

Porter, M. E. (1990). The competitive advantage of nations. Nueva York: Free Press.

Quivy, R., \& Van Campenhoudt, L. (2006). Manual de investigación en ciencias sociales. México: Limusa.

Rego, P. (2003). Minicadenas productivas: una alternativa para el desarrollo regional. Bogotá: Cinset.
Samuelson, P., \& Northdaus, W. (2002). Economía (17a ed.). México: McGraw-Hill.

Sánchez, A. (2014). Crisis en la frontera. Documentos de trabajo sobre Economía Regional ${ }^{\circ} 197$. Bogotá: Banco de la República. Recuperado de http://www. banrep.gov.co/docum/Lectura_finanzas/pdf/dtser_197.pdf Seldon, A., \& Pennance, F. (1990). Diccionario de economía. España: Oikos.

Serna, H. (2000). Planificación y gestión estratégica. Colombia: Legis.

Shumpeter, J. A. (1976). Teoría del desenvolvimiento económico. México: Fondo de Cultura Económica.

Soto, R., \& Bergoeing, R. (1998). Una evaluación preliminar del impacto económico de $\mathrm{El} \mathrm{Te}$ niente en la sexta Región. Chile: Hades Georgetown University. Stiglitz, J. E. (2003). Los felices 90: La semilla de la destrucción. Madrid: Taurus. 